\title{
Software systems for operation, control, and monitoring of the EBEX instrument
}

Michael Milligan, ${ }^{a}$ Peter Ade, ${ }^{b}$ François Aubin, ${ }^{c}$ Carlo Baccigalupi, ${ }^{d}$ Chaoyun Bao, ${ }^{a}$ Julian Borrill, ${ }^{e}$ Christopher Cantalupo, ${ }^{e}$ Daniel Chapman,${ }^{f}$ Joy Didier,${ }^{f}$ Matt Dobbs,${ }^{c}$ Will Grainger, ${ }^{b}$ Shaul Hanany, ${ }^{a}$ Seth Hillbrand, ${ }^{f}$ Johannes Hubmayr, ${ }^{g}$ Peter Hyland, ${ }^{c}$ Andrew Jaffe, ${ }^{h}$ Bradley Johnson, ${ }^{i}$ Theodore Kisner, ${ }^{e}$ Jeff Klein, ${ }^{a}$ Andrei Korotkov, ${ }^{j}$ Sam Leach, ${ }^{d}$ Adrian Lee, ${ }^{i}$ Lorne Levinson, ${ }^{k}$ Michele Limon,${ }^{f}$ Kevin MacDermid, ${ }^{c}$ Tomotake Matsumura, ${ }^{l}$ Amber Miller,${ }^{f}$ Enzo Pascale, ${ }^{b}$ Daniel Polsgrove, ${ }^{a}$ Nicolas Ponthieu, ${ }^{m}$ Kate Raach,${ }^{a}$ Britt Reichborn-Kjennerud,${ }^{f}$ Ilan Sagiv, ${ }^{a}$ Huan Tran,${ }^{i}$ Gregory S. Tucker, ${ }^{j}$ Yury Vinokurov,${ }^{j}$ Amit Yadav, ${ }^{n}$ Matias Zaldarriaga, ${ }^{n}$ Kyle Zilic ${ }^{a}$

${ }^{a}$ University of Minnesota School of Physics and Astronomy, Minneapolis, MN 55455;

${ }^{b}$ Cardiff University, Cardiff, CF24 3AA, United Kingdom;

${ }^{c}$ McGill University, Montréal, Quebec, H3A 2T8, Canada;

${ }^{d}$ Scuola Internazionale Superiore di Studi Avanzati, Trieste 34151, Italy;

${ }^{e}$ Lawrence Berkeley National Laboratory, Berkeley, CA 94720;

${ }^{f}$ Columbia University, New York, NY 10027;

${ }^{g}$ National Institute of Standards and Technology, Boulder CO 80303;

${ }^{h}$ Imperial College, London, SW7 2AZ, England, United Kingdom;

${ }^{i}$ University of California, Berkeley, Berkeley, CA 94720;

${ }^{j}$ Brown University, Providence, RI 02912;

${ }^{k}$ Weizmann Institute of Science, Rehovot 76100, Israel;

${ }^{l}$ California Institute of Technology, Pasadena, CA 91125;

${ }^{m}$ Institut d'Astrophysique Spatiale, Universite Paris-Sud, Orsay, 91405, France;

${ }^{n}$ Institute for Advanced Study, Princeton, NJ 08540

\begin{abstract}
We present the hardware and software systems implementing autonomous operation, distributed real-time monitoring, and control for the EBEX instrument. EBEX is a NASA-funded balloon-borne microwave polarimeter designed for a 14 day Antarctic flight that circumnavigates the pole.

To meet its science goals the EBEX instrument autonomously executes several tasks in parallel: it collects attitude data and maintains pointing control in order to adhere to an observing schedule; tunes and operates up to 1920 TES bolometers and 120 SQUID amplifiers controlled by as many as 30 embedded computers; coordinates and dispatches jobs across an onboard computer network to manage this detector readout system; logs over $3 \mathrm{GiB} /$ hour of science and housekeeping data to an onboard disk storage array; responds to a variety of commands and exogenous events; and downlinks multiple heterogeneous data streams representing a selected subset of the total logged data. Most of the systems implementing these functions have been tested during a recent engineering flight of the payload, and have proven to meet the target requirements.

The EBEX ground segment couples uplink and downlink hardware to a client-server software stack, enabling real-time monitoring and command responsibility to be distributed across the public internet or other standard computer networks. Using the emerging dirfile standard as a uniform intermediate data format, a variety of front end programs provide access to different components and views of the downlinked data products. This distributed architecture was demonstrated operating across multiple widely dispersed sites prior to and during the EBEX engineering flight.
\end{abstract}

Keywords: CMB, millimeter-wave telescopes, flight control systems, ballooning, data handling

Software and Cyberinfrastructure for Astronomy, edited by Nicole M. Radziwill, Alan Bridger,

Proc. of SPIE Vol. 7740, 774007 - @ 2010 SPIE · CCC code: 0277-786X/10/\$18 · doi: 10.1117/12.857583 


\section{INTRODUCTION}

\subsection{Science goals}

The E and B EXperiment (EBEX) is balloon-borne microwave polarimeter designed to study the polarization of the cosmic microwave background (CMB) and the foreground emission of thermal dust in our galaxy. ${ }^{1,2}$ These measurements will: detect or constrain the primordial B-mode polarization of the CMB, a predicted signature of gravity waves produced by cosmic inflation; $;^{3,4}$ characterize the polarized foreground dust emission, which is a necessary step in determining the CMB B-mode signal; ${ }^{5,6}$ and measure the predicted effect of gravitational lensing on the CMB. ${ }^{7}$ The science goals of EBEX are described more fully in other publications. ${ }^{1,2,8}$

\subsection{Instrument description}

The EBEX instrument consists of a 1.5 meter clear aperture Gregorian-type telescope that feeds a cryogenic receiver, all of which are mounted on the inner frame of the EBEX gondola. Pointing control is maintained by driving the inner frame in elevation, while a pivot and reaction wheel turn the outer frame azimuthally relative to the balloon flight line. Attitude sensors including a sun sensor, star cameras, differential GPS, gyroscopes, magnetometer, and clinometers are mounted as appropriate on the inner and outer frames. The flight computers, Attitude Control System (ACS) crate, and disk storage pressure vessels are mounted on the outer frame. Inside the cryostat reimaging optics focus the input radiation onto two focal planes each carrying up to 960 transition edge sensor (TES) bolometers, up to 1920 total bolometers. A polarimetric system, consisting of a half wave plate $(\mathrm{HWP})^{9}$ spinning on a superconducting magnetic bearing ${ }^{10}$ and a wire grid, modulates polarization information into the phase and amplitude of the component of the radiation intensity at the focal plane corresponding to four times the HWP rotation frequency. ${ }^{11}$ The TES are read out through SQUID amplifiers via a frequency domain multiplexing scheme that connects up to 16 TES to each SQUID. The SQUIDs in turn are connected in groups of four to digital frequency-domain multiplexing readout (DfMux) boards. ${ }^{12}$ The design of the EBEX instrument is detailed elsewhere, ${ }^{2,8,13}$ and the bolometer readout system is described in Hubmayr et al ${ }^{14}$ and Aubin et al. ${ }^{15}$ EBEX completed a 13 hour engineering flight from Ft. Sumner, New Mexico in June 2009. In this paper we describe the software and data flow architecture that make up the EBEX control and monitoring systems.

\subsection{Computing and system control overview}

In order to meet the science goals, EBEX autonomously executes several tasks in parallel.

The instrument maintains real-time pointing control to better than the $0.5^{\circ}$ requirement and logs sufficient data from the pointing sensors to allow post-flight pointing reconstruction to better than the $9^{\prime \prime}$ requirement. The pointing system can realize several predefined instrument scan modes, as well as drift, slew, and coordinate tracking motions. The two redundant flight computers (see Sec. 2.1) execute all pointing actions synchronously, with a watchdog card selecting the less-recently rebooted computer to control the instrument. The pointing system is discussed in detail by Reichborn-Kjennerud. ${ }^{16}$

Both SQUIDs and TES bolometers periodically require active tuning, such as during cycling of the sub-Kelvin adsorption refrigerators. ${ }^{17}$ This instrument reads out up to 1792 of the 1920 bolometers, multiplexed through 112 SQUIDs, operated by 28 DfMux boards. These setup and tuning operations are managed over the gondola Ethernet network by the flight computers, as discussed in Sec. 2.2.

Bolometers are read out at $190.73 \mathrm{~Hz}$ 16-bit samples. Depending on the multiplexing level each DfMux board reads out between 32 and 64 bolometers, producing a data stream of between 21 and 42 kilobytes/s, or 2.1 to 4.2 gigabytes per hour for the full complement of 28 boards. The ACS generates an additional data stream of approximately $20 \mathrm{~KB} / \mathrm{s}$ (70 megabytes per hour), and the angular encoders on the rotating HWP produce a combined $21 \mathrm{~KB} / \mathrm{s}(75 \mathrm{MB} / \mathrm{h})$. This output data is transferred over the ethernet network to the flight computer and logged to disk. Consequently for a 14 day flight the onboard disk array must provide over 1.5 terabytes total storage per redundant copy written. The storage system is discussed in Sec. 2.3.

In addition to planned housekeeping operations, the possibility of unplanned events demands that EBEX possess the ability to respond to some exogenous contingencies, that sufficient operational data be downlinked to enable human diagnosis of unexpected conditions, and that the telecommanding interface be flexible enough 
to exercise the full range of recovery options available in the flying hardware. The necessary downlink (Sec. 2.4) is provided by a $1 \mathrm{Mbit} / \mathrm{s}$ line-of-sight (LOS) transmitter available for roughly the first day of flight, and a much slower TDRSS satellite relay afterwards. The telecommanding uplink relies on satellite relay or an HF-band LOS transmission, and in practice is limited to less than ten 15-bit command tokens per second.

All of the above activities can be triggered from the ground via uplinked commands, as well as scheduled via onboard schedule files. The scheduling system operates in local sidereal time, allowing planned observations to account for the motion of the balloon in longitude, which cannot be precisely known in advance. Within the limits of the underlying operating system, actions can be scheduled arbitrarily far in the future. Uplinked commands can select between alternative stored schedules.

The communications infrastructure of the Columbia Scientific Balloon Facility (CSBF) provides the LOS downlink signal at the launch site, and provides connections to satellite-based telemetry and telecommanding via the Operations Control Center in Palestine, Texas. ${ }^{18}$ During a long duration balloon flight, many collaboration personnel will be positioned at the launch site, while other collaborators may be geographically dispersed. To support this scenario the EBEX ground segment couples uplink and downlink hardware to a client-server software stack (see Sec. 2.5 and Fig. 2). The full high rate LOS data stream is available at multiple client workstations at the launch site, and portions of this data can be made available via the public internet for remote real-time examination. Likewise telecommanding is forwarded over network links to the EBEX ground station and CSBF uplink.

To meet the reliability and development time requirements of this project we use commercially available hardware and existing software whenever practical. With the exception of the FPGA-based DfMux and ACS boards, onboard computers and networking hardware are available industrial embedded models which we have qualified in thermal and vacuum conditions approximating balloon flight. The ACS, many aspects of the gondola and pointing system design, and several components of the software chain described here originate with the BLAST project, ${ }^{19}$ and are described by Wiebe. ${ }^{20}$ The housekeeping system makes extensive use of embedded monitoring boards ${ }^{21}$ originally developed for the ATLAS experiment at CERN. ${ }^{22,23}$

\section{SYSTEMS}

The EBEX gondola comprises several subsystems of networked components, with the flight computer crate acting as the point of intersection.

An Ethernet network of industrial ring switches ${ }^{24}$ connects the flight computers, disk storage system, bolometer readout boards, HWP encoder readouts, sun sensor, and star camera. This network is shown in Fig. 1. The use of ring switches provides resilience to network breaks or failure of a single switch. Optical fiber connections are used where electrical isolation is necessary.

The GPS receiver, multiple actuators, and the CSBF support package (which includes the commanding uplink and low rate satellite telemetry) communicate directly with the flight computers via serial ports. Additional sensors and controls connect directly to hardware in the ACS crate. The ACS communicates with the flight computers via a custom bidirectional bus termed the "E-bus." 16,20

Housekeeping monitoring and control is handled by custom boards equipped with embedded monitoring boards, ${ }^{23}$ which are connected by a Controller Area Network ${ }^{25}$ bus (CANbus). The flight computers communicate with this network via Kvaser USB-CANbus adapters. ${ }^{26}$

Because the housekeeping system, ACS, and bolometer readouts are asynchronous, all systems embed in their data streams a common timestamp using EBEX "ticks" which is recorded for post-flight alignment. The systems maintain a relative synchronization of $\sim 10 \mu$ s by resynchronizing to an onboard precision clock every $164 \mathrm{~ms}$. The time servers broadcast synchronization messages onto the CANbus, and distribute timing data to the DfMux boards and ACS via an RS-485 serial link that does not connect to the flight computers. The housekeeping and timing subsystems are described in Sagiv et al. ${ }^{13}$ 


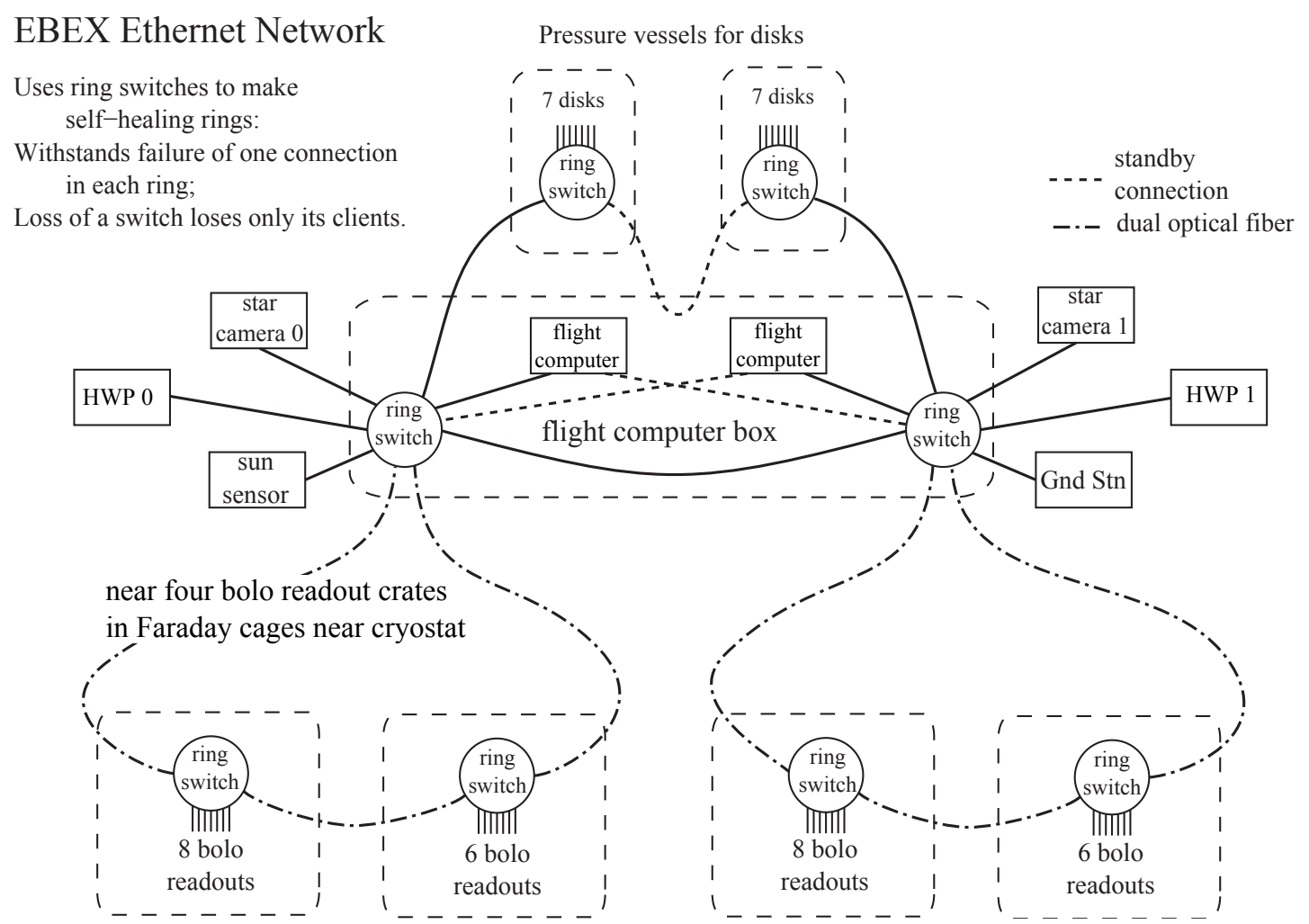

Figure 1. Configuration of the EBEX gondola ethernet network planned for the long duration Antarctic flight.

\subsection{Flight control program - fcp}

The flight computer crate contains two Ampro single board computers, ${ }^{27}$ each configured with a $1.0 \mathrm{GHz}$ Celeron processor, $256 \mathrm{MiB}$ RAM, and a $1 \mathrm{~GB}$ solid state flash disk module. The module stores the computer operating system, currently Debian GNU/Linux 4.0 ${ }^{28}$ with Linux kernel 2.6.18 and additional modular drivers for the ACS E-bus and USB-CANbus adapter. The flight control program $f c p$ resides on the flash module as well, which the operating system is configured to run immediately after the computer boots.

$f c p$ is a derivative of the BLAST experiment's $m c p,{ }^{20}$ and preserves its overall architecture as a monolithic program running multiple concurrent, event-driven threads, with a main loop handling pointing, frame generation, and data logging clocked to the E-bus. We have added code modules implementing control and readout of the DfMux boards, housekeeping via the CANbus, storage to the networked disk storage array, and the downlink scheme discussed below. Other modules have been modified as needed.

Flight computer redundancy is implemented via a watchdog card connected to the IEEE 1284 parallel port of each computer. In nominal operation the $f c p$ WatchDog thread toggles a pin on the parallel port at $25 \mathrm{~Hz}$. If this action ceases for more than a configurable length of time, a fault is inferred. The watchdog card will power cycle the faulty computer and switch control to the other computer. Besides crashes in the software or hardware of the flight computer, $f c p$ can programmatically trigger this sequence of events by terminating the WatchDog thread in response to certain error conditions. The identity of the computer in control is communicated to both flight computers via the E-bus, and recorded as the incharge variable. During the North American engineering flight dataset the value of this variable changes only once, at 8:19 UTC, due to an intentional pre-flight reboot of the then-active flight computer. This indicates that there were no such reboots of the in-charge flight computer between launch at 14:01 UTC and termination after 03:18 UTC.

\subsection{Distributed networked bolometer readout architecture}

Each DfMux readout board combines analog signal processing hardware with an FPGA implementing digital signal processing modules and a soft CPU running an embedded Linux distribution. The DfMux hardware is 


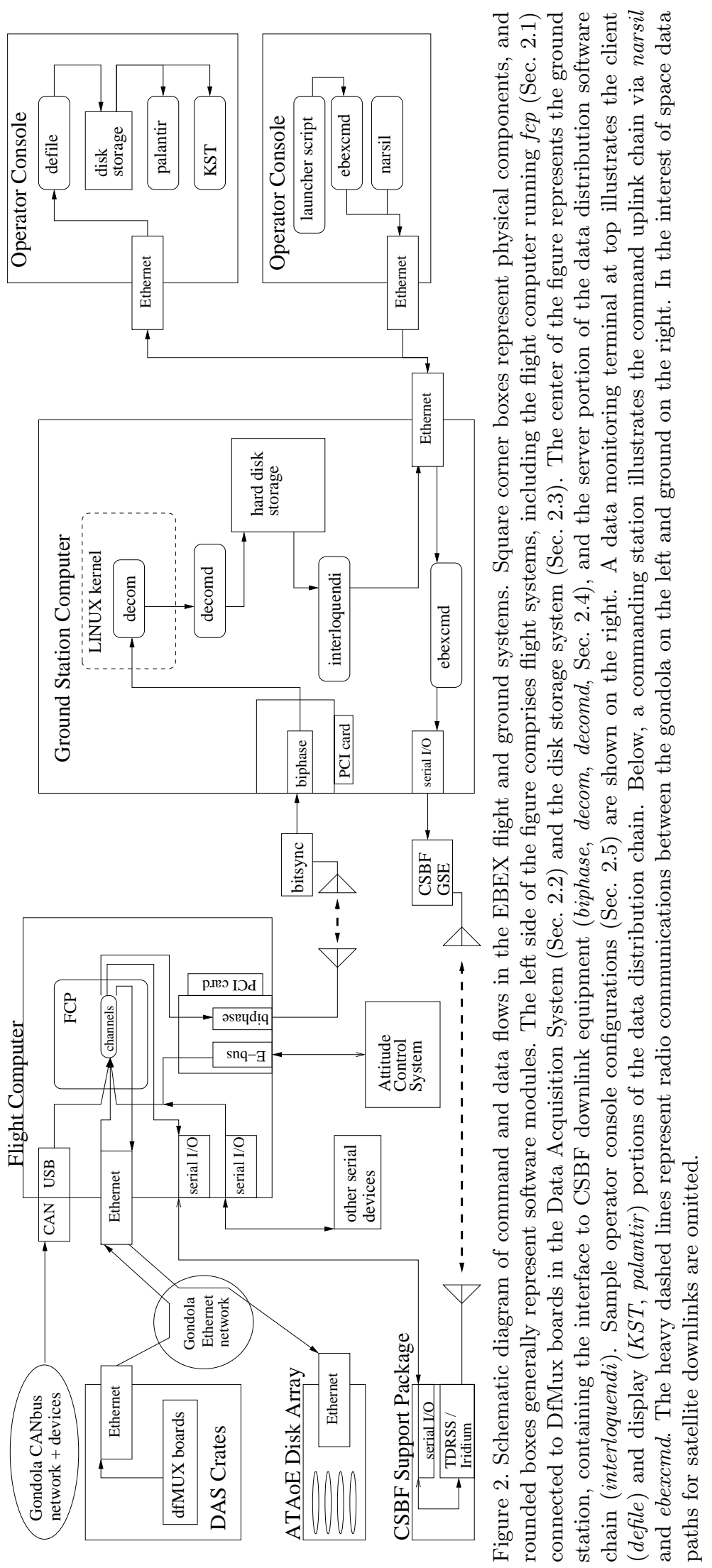

Proc. of SPIE Vol. $7740774007-5$ 
described in detail by Dobbs et al. ${ }^{12}$ Operations comprising the setup, tuning, and maintenance of the detectors and readout system are controlled by the flight computer via requests over the Ethernet network, and readout data are returned over the same network.

Low level operations are exposed via small programs in the DfMux firmware implementing the Common Gateway Interface. ${ }^{29}$ More complex algorithms are invoked as jobs through an interface called "Algorithm Manager," which passes data using JavaScript Object Notation. ${ }^{30}$ On each DfMux board a program, implemented by code in a subset of the Python language ${ }^{31}$ listens on a network port for requests to start, stop, or collect the output of jobs. Because of memory and CPU constraints in the embedded environment, no more than two jobs may run at a time on each board. In $f c p$ the algMan module maintains queues of pending and running jobs and attempts to run all requested jobs as soon as possible, while ensuring that on a per-board basis all jobs are run in the order requested. To the rest of $f c p$, algMan exposes routines to trigger algorithm requests to a single board. It also provides a higher level interface based on stored parameter files. In these files sets of algorithm parameters are defined on a per-SQUID basis. After commanding $f c p$ to parse one of the stored files, algMan will respond to these high-level commands by dispatching algorithm requests for the corresponding operation for each SQUID defined in the parameter file.

Regardless of the method of invocation, requested operations will produce output strings in the JavaScript Object Notation format which are returned to algMan. These strings, generically termed "algorithm results," are logged to disk and added to the file downlink system queue.

DfMux boards output data samples by broadcasting User Datagram Protocol ${ }^{32}$ packets to a multicast address over the Ethernet network. Each packet is 1428 bytes and consists of a header and 13 frame structures. In the case of the bolometer readout boards in the configuration flown in the 2009 engineering flight, with 8 bolometer per SQUID multiplexing (32 total bolometer channels per board) these frames contain a timestamp and one 16-bit sample for each of the 32 channels recorded at the corresponding time. For a $190.73 \mathrm{~Hz}$ sample rate each board broadcasts packets at $14.67 \mathrm{~Hz}$. Within each bolometer readout crate, the DfMux boards are synchronized to a common $25 \mathrm{MHz}$ oscillator so that the bolometers for all boards in the crate are sampled at the same time.

In $f c p$ the UDPS_Listener packet reader thread listens on the multicast address. Each packet is inspected to determine its origin, and the pdump module writes it to disk in a packet dump (.pdump) file corresponding to the originating board. The .pdump files are rotated every 15 minutes to limit the maximum file size produced. Fig. 3 demonstrates the performance of this readout system for a typical readout board. Excluding a brief period around 17:35 UTC when the boards were commanded to reboot during a SQUID tuning procedure, no board is missing more than 65 packets from the logged packet data, for a loss rate of $<0.01 \%$. Testing on the ground shows that under simulated load equivalent to the full planned complement of 28 boards, loss rates remain similarly low. 11 of the 12 bolometer readout boards were synchronized to the common oscillators in their respective crates for the entire flight. The twelfth board was left unsynchronized due to a misconfigured startup script.

Two DfMux boards are also used to read the optical angular encoder on the HWP. They each sample a single channel at $3.052 \mathrm{KHz}$. Each HWP encoder packet contains 416 samples, and thus each board broadcasts packets at $7.34 \mathrm{~Hz}$. The structure of the bolometer readout packets is reused for the HWP encoder readout, so the same code processes both types of packet stream.

The code defining the packet format is written in portable $\mathrm{C}$ that is compiled into the packet streamer program onboard the DfMux CPU, UDPS_Listener, and the standalone parser program used to extract data from packet streams and saved dumps.

\subsection{ATAoE onboard storage}

EBEX will fly with over 3 terabytes of hard disk storage. This allows the flight computers to write two redundant copies of all data produced in flight to separate disks. We use the ATA over Ethernet (ATAoE) protocol ${ }^{33}$ in order to implement the onboard disk storage array. Ethernet has several attractive features. It provides a manyto-many topology so that redundant disks can be provided without foreknowledge of which flight computer will need one. It is physically straightforward to route signals from the flight computer crate in vacuum into the 


\section{DfMux 50 post-flight timestamp flags}

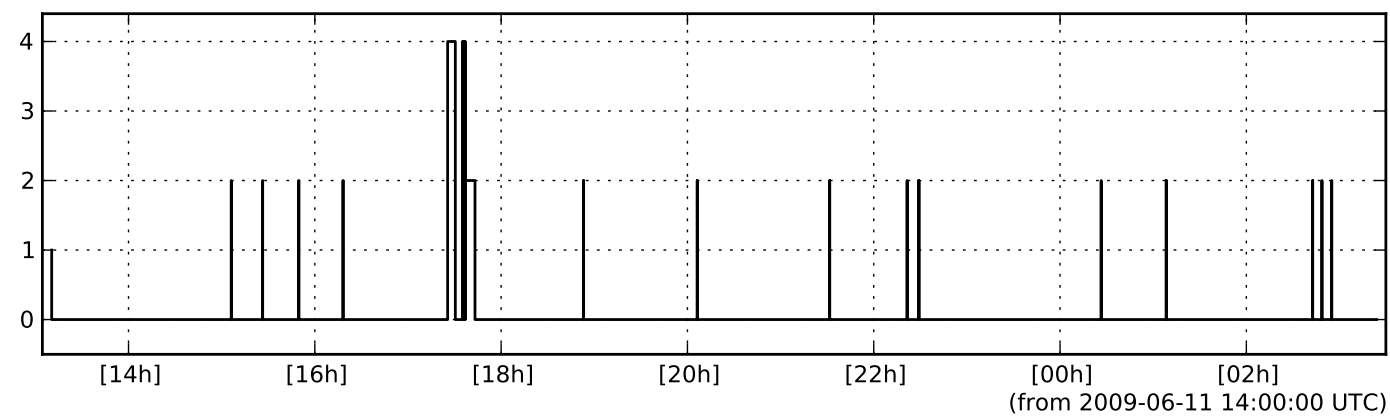

Figure 3. Synchronization flags for a typical bolometer readout board during the 2009 flight. The flag values indicate: 0 - sample present and synchronized; 1 - padding at ends; 2 - missing data; 4 - wrong sample rate. The anomalous behavior around 17:35 UTC corresponds to a commanded reboot of the DfMux boards. Most of the isolated spikes to state 2 indicate single packets missing from the recorded data stream, 20 in total for this board. Otherwise for this board data samples were logged for the entire flight, and those samples were synchronized to the common oscillator.

pressure vessels holding hard disks. Finally, Ethernet is already in use onboard so it avoids adding an additional networking technology. Drivers for the ATAoE protocol are a standard part of the Linux kernel.

As shown in Fig. 1, the disk drives are divided between two pressure vessels. Each vessel contains a ring switch, a passive backplane for power and signal distribution, and up to seven 2.5" laptop disk drives mounted on ATAoE blades. ${ }^{34}$ Each blade is connected independently to the ethernet ring switch. In $f c p$ the EBEX_AOE module abstracts detection, setup and low-level management of the array. Disk usage is flagged in non-volatile memory present on each blade to ensure that the two flight computers do not attempt to simultaneously mount the same disk. This module will only present as available disks which are not already in use and which have sufficient free space remaining. The aoeMan module adds an additional layer of abstraction, allowing $f c p$ code to request file operations without any detailed knowledge of the disk array. It mounts disks as needed to supply the requested free space, and translates filenames to correspond with the correct mount points in the filesystem namespace.

\subsection{Downlink and data logging}

$f c p$ produces a $1 \mathrm{Mbit} / \mathrm{s}$ biphase encoded output data stream, suitable for transmission over the CSBF-provided line-of-sight downlink. This stream combines all output channels of the ACS and housekeeping systems, packet data streams from five selectable DfMux boards, and a file downlink system called filepig, used to retrieve algorithm results, diagnostic logs, and other irregularly formatted data. At the launch site the EBEX ground station uses a commercial bit synchronizer, custom decommutator card, and the decomd software to decode and store this data stream to disk.

As detailed in Fig. 4, the downlink stream is composed of 1248 byte frames generated at $100 \mathrm{~Hz}$. These are grouped into superframes of 20 frames. Each frame begins with a sync word and counters, followed by channel data. Each 2-byte word of channel data can either contain samples of a "fast channel" at $100 \mathrm{~Hz}$, or have 20 "slow channels" multiplexed over the superframe at $5 \mathrm{~Hz}$. In the 2009 engineering flight, this channel data totalled 194 bytes per frame, encoding 59 fast channels and 480 slow channels. This channel data is also logged to disk onboard the gondola.

The remaining space in each frame (1048 bytes, after overhead, for the 2009 flight configuration) is aggregated across the superframe and used to transfer DfMux readout packets and filepig data blocks. In $f c p$ this format is defined by the "Biphase marshaler" module, which accepts data from UDPS_Listener and filepig and assembles the superframe data area. Every $200 \mathrm{~ms}$ the $f c p$ downlink code queries the marshaler for an assembled data area to incorporate into the transmitted frames.

The marshaler uses fixed slots in the superframe to provision a deterministic bandwidth to each downlinked data stream, and to ensure that if one frame is lost or corrupted, data in the surrounding frames can still be correctly reassembled. UDPS_Listener, described above, passes whole packets, and thus requires 1428-byte slots. 


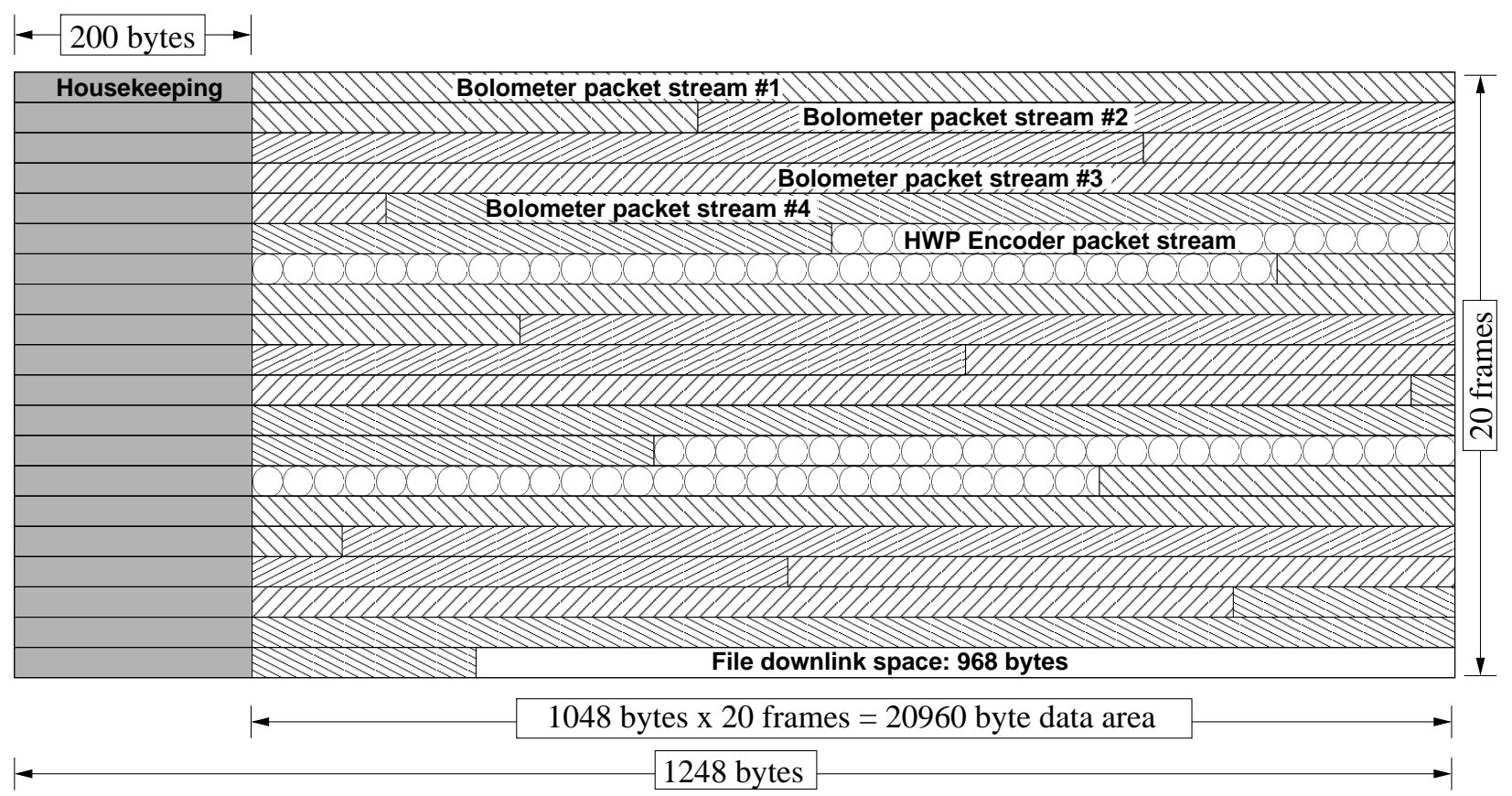

Figure 4. Schematic of the line-of-sight downlink superframe discussed in Sec. 2.4. This structure is repeated at $5 \mathrm{~Hz}$ over the $1 \mathrm{Mbit} / \mathrm{s}$ transmitter. The horizontal rows indicate the 20 individual 1248 byte frames, transmitted at $100 \mathrm{~Hz}$. Each frame starts with 200 bytes of header and housekeeping channel data. The remaining 1048 bytes in each frame is aggregated across the superframe to form a 20960 byte data area. 14 slots of 1428 bytes each are allotted for DfMux packets and are grouped into five logical streams (denoted here by matching hatch patterns), accomodating the complete data output of four bolometer readout boards and one HWP encoder readout board. The final 968 bytes of the superframe is used by the filepig file downlink system.

In $200 \mathrm{~ms}$ a bolometer readout board produces on average 2.93 packets, and a HWP encoder board produces 1.47. Thus a group of three slots for bolometer readout or two slots for encoder readout yields a stream with adequate capacity to downlink the entire packet data output of a DfMux board. With 14 slots, streams are defined to downlink the output of four bolometer readout boards and one HWP encoder board. Uplinked commands select which five boards out of the total complement are allotted a downlink stream.

filepig, so named because it allows files to "piggyback" on a frame-based protocol, claims the odd-sized chunk of space at the end of the data area after packet streams have been allocated. It exposes an interface by which $f c p$ code may queue the filenames of data objects already written to disk. Files are broken into chunks together with minimal header and error detection data and downlinked. Support exists, presently unused, to plug in transformations for more robust error correction or data compression, and to resend corrupted data in response to uplinked commands. For the engineering flight 968 bytes per superframe were left for the filepig data chunk, providing about $4.2 \mathrm{~KB} / \mathrm{s}$ file downlink bandwidth. Over the 13 hour flight 10898 files totalling 61 MB were retrieved.

\subsection{Ground tools and architecture}

The BLAST telemetry chain ${ }^{20}$ is employed largely unmodified on the ground. As illustrated in Fig. 2, the biphase encoded bitstream is converted back into data frames in the Ground Station computer and logged to disk. The interloquendi server permits clients to fetch streams of frames remotely via TCP/IP connections. defile then decodes the channel data in these frames into dirfile ${ }^{35}$-format data files. Front end programs such as palantir and $K S T^{36}$ allow real-time display of the streamed channels.

To this EBEX adds support in the frame handling code for the superframe data area, and support in defile for extracting packet streams and downlinked files from those frames. These additional data products are written alongside the channel-based data on each connecting client workstation. Scripts employing the parser program 
automate the production of bolometer and HWP encoder time streams in dirfile format from extracted . pdump files.

Time streams can be displayed in real-time using either KST or Python tools that understand the dirfile format. The EBEX Alignment Tools is a suite of programs for further processing these streams, including interpolation and alignment to a common sample rate and timing, decoding the HWP angular encoder signal to HWP position, and template-based removal of the HWP rotation signal from bolometer timestreams.

We have also written a Python/TK front end to the Algorithm Manager system. By monitoring the names of the files downlinked through filepig, it is possible to select those corresponding to algorithm result strings. Parsing these files permits display on a board-by-board basis, in close to real time, of the job execution activity occurring in the readout system DfMux boards. A dashboard interface presents selected information from each board using labels and color coding, and the user can select individual boards or jobs for more detailed display. This front end provides immediate visual feedback on complex operations, such as detector system tuning, that entail parallel execution of a sequence of jobs on each bolometer readout board.

ebexcmd accepts $f c p$ commands in textual format, which it can either relay to a listening ebexcmd over a network connection, or convert to the binary representation suitable for transmission over CSBF uplink hardware. Commands can therefore be generated on any host permitted to connect to the ground station, and those commands will then be uplinked. Commands are most commonly selected through the narsil front end, but are also generated by Python scripts and may even be entered manually from a command line.

This ground infrastructure provides network transparency in both data distribution and commanding, allowing flight operators to monitor and control the instrument from an arbitrary number of networked workstations. During the 2009 integration campaign and flight, this system routinely connected as many as ten client workstations over the private internal network at the New Mexico launch site. Late in the flight line-of-sight communications were only possible from the downrange station in Arizona, and commands were successfully relayed from the launch site through the downrange ground station ebexcmd. Streaming of frame data via interloquendi from the downrange station to the launch site, and from the launch site to collaborators at their home institutions, worked only intermittently due to bandwidth constraints at the launch site.

\section{CONCLUSION}

EBEX combines a large format bolometer array, and the correspondingly large data volume, with a complex readout system architecture. As a result, EBEX solves for a balloon flight environment problems in data handling, communications, and control that are typically associated with ground based observatories. The required 3 terabyte in-flight storage capacity is achieved using a high speed gondola ethernet network and networked disk storage arrays. The readout system is controlled from a central flight computer using a custom distributed job control scheduler, and it is monitored by extending a frame-oriented telemetry system to support asynchronous packet streams and event-driven downlink of arbitrary data in files. On the ground, a networked real-time data distribution and command relay architecture allows shared monitoring and control of the instrument.

\section{ACKNOWLEDGMENTS}

EBEX is supported by NASA through grants number NNG04GC03G, NNG05GE62G, NNX08AG40G, and NNX07AP36H. Additional support comes from the National Science Foundation through grant number AST 0705134, the French Centre national de la recherche scientifique (CNRS), and the UK Science and Technology Facilities Council (STFC). This project makes use of the resources of the Minnesota Supercomputing Institute and of the National Energy Research Scientific Computing Center (NERSC), which is supported by the office of Science of the U.S. Department of Energy under contract No. DE-AC02-05CH11231. The McGill authors acknowledge funding from the Canadian Space Agency, Natural Sciences and Engineering Research Council, Canadian Institute for Advanced Research, Canadian Foundation for Innovation and Canada Research Chairs program.

We thank the Columbia Scientific Balloon Facility for their energetic support. We gratefully acknowledge the efforts of our colleagues in the BLAST project that produced the foundation for much of this work. 


\section{REFERENCES}

[1] Oxley, P. et al., "The EBEX experiment," Infrared Spaceborne Remote Sensing XII 5543(1), 320-331, SPIE (2004).

[2] Grainger, W. et al., "EBEX: the E and B Experiment," Millimeter and Submillimeter Detectors and Instrumentation for Astronomy IV 7020(1), 70202N, SPIE (2008).

[3] Seljak, U. and Zaldarriaga, M., "Signature of Gravity Waves in the Polarization of the Microwave Background," Physical Review Letters 78, 2054-2057 (Mar. 1997).

[4] Hu, W., Seljak, U., White, M., and Zaldarriaga, M., "Complete treatment of CMB anisotropies in a FRW universe," Phys. Rev. D 57, 3290-3301 (Mar. 1998).

[5] Brandt, W. N., Lawrence, C. R., Readhead, A. C. S., Pakianathan, J. N., and Fiola, T. M., "Separation of foreground radiation from cosmic microwave background anisotropy using multifrequency measurements," ApJ 424, 1-21 (Mar. 1994).

[6] Baccigalupi, C., "Cosmic microwave background polarisation: foreground contrast and component separation," New Astronomy Review 47, 1127-1134 (Dec. 2003).

[7] Zaldarriaga, M. and Seljak, U., "Gravitational lensing effect on cosmic microwave background polarization," Phys. Rev. D 58, 023003-+ (July 1998).

[8] Reichborn-Kjennerud, B. et al., "EBEX: a balloon-borne CMB polarization experiment," Millimeter, Submillimeter, and Far-Infrared Detectors and Instrumentation for Astronomy V 7741, SPIE (2010).

[9] Hanany, S., Hubmayr, J., Johnson, B. R., Matsumura, T., Oxley, P., and Thibodeau, M., "Millimeter-wave achromatic half-wave plate," Appl. Opt. 44, 4666-4670 (Aug. 2005).

[10] Hull, J. R., Hanany, S., Matsumura, T., Johnson, B., and Jones, T., "Characterization of a high-temperature superconducting bearing for use in a cosmic microwave background polarimeter," Superconductor Science and Technology 18(2), S1 (2005).

[11] Johnson, B. R., MAXIPOL: A bolometric, balloon-borne experiment for measuring the polarization anisotropy of the cosmic microwave background radiation, $\mathrm{PhD}$ thesis, University of Minnesota (2004).

[12] Dobbs, M., Bissonnette, E., and Spieler, H., "Digital Frequency Domain Multiplexer for MillimeterWavelength Telescopes," IEEE Transactions on Nuclear Science 55, 21-26 (2008).

[13] Sagiv, I. et al., "The EBEX cryostat and supporting electronics," to appear in Proceedings of the Twelth Marcel Grossmann Meeting ${ }^{37}$ (2010).

[14] Hubmayr, J., Aubin, F., Bissonnette, E., Dobbs, M., Hanany, S., Lee, A. T., MacDermid, K., Meng, X., Sagiv, I., and Smecher, G., "Design and characterization of TES bolometers and SQUID readout electronics for a balloon-borne application," Millimeter and Submillimeter Detectors and Instrumentation for Astronomy IV 7020(1), 70200J, SPIE (2008).

[15] Aubin, F. et al., "SQUID-based multiplexed readout electronics and TES bolometer array during an engineering flight of the EBEX stratospheric balloon," Millimeter, Submillimeter, and Far-Infrared Detectors and Instrumentation for Astronomy V 7741, SPIE (2010).

[16] Reichborn-Kjennerud, B., Building and Flying the E and B Experiment to Measure the Polarization of the Cosmic Microwave Background, PhD thesis, Columbia University (2010).

[17] Chase, S., [Two-stage sub-Kelvin ${ }^{3}$ He cooler], Chase Research Cryogenics Ltd., 140 Manchester Road, Sheffield S10 5DL, England.

[18] NASA Columbia Scientific Balloon Facility, LDB Science Enclosures (2010).

[19] Pascale, E. et al., "The Balloon-borne Large Aperture Submillimeter Telescope: BLAST," ApJ 681, 400414 (July 2008).

[20] Wiebe, D. V., BLAST: A Balloon-borne, Large-aperture, Submillimetre Telescope, PhD thesis, University of Toronto (Feb. 2009).

[21] Boterenbrood, H. and Hallgren, B., "The development of the Embedded Local Monitor Board (ELMB)," in [PROCEEDINGS of the Ninth Workshop on Electronics for LHC Experiments], 9th Workshop on Electronics for LHC Experiments, 331-334 (2003).

[22] Boterenbrood, H., Burckhart, H. J., Cook, J., Filimonov, V., Hallgren, B., Heubers, W., Khomoutnikov, V., Ryabov, Y., and Varela, F., "Design and Implementation of the ATLAS Detector Control System," IEEE Transactions on Nuclear Science 51, 495-501 (June 2004). 
[23] Boterenbrood, H., Burckhart, H., and Kvedalen, H., "The Embedded Local Monitor Board (ELMB) in the LHC front-end I/O control system," in [PROCEEDINGS of the Seventh Workshop on Electronics for LHC Experiments], 7th Workshop on Electronics for LHC Experiments, 325-330 (2001).

[24] Sixnet LLC, http://sixnet.com, EtherTRAK Industrial Ethernet Real-Time Ring switch. various models.

[25] ISO 11898-1:2003, [Road vehicles - Controller area network (CAN) - Part 1: Data link layer and physical signalling], International Organization for Standardization, Geneva, Switzerland (2003).

[26] Kvaser Inc., http://www.kvaserinc.com, Leaf SemiPro HS.

[27] Ampro ADLINK Technology, Inc., http://ampro.com, MightyBoard 800.

[28] Debian Project, http://debian.org, Debian GNU/Linux 4.0 ("Etch").

[29] Robinson, D. and Coar, K., "The Common Gateway Interface (CGI) Version 1.1." RFC 3875 (Informational) (Oct. 2004).

[30] Crockford, D., "The application/json Media Type for JavaScript Object Notation (JSON)." RFC 4627 (Informational) (July 2006).

[31] van Rossum, G. et al., Python Language Reference. http://www.python.org/.

[32] Postel, J., "User Datagram Protocol." RFC 768 (Standard) (Aug. 1980).

[33] Hopkins, S. and Coile, B., ATA over Ethernet Specification. The Brantley Coile Company, Inc. (Feb. 2009).

[34] CORAID Inc., http://www.coraid.com, EtherDrive storage solutions.

[35] Wiebe, D. V., Dirfile Standards. http://getdata.sourceforge.net.

[36] Netterfield, B. et al., KST. KDE Project, http://kst.kde.org/.

[37] Damour, T., Jantzen, R. T., and Ruffini, R., eds., [Proceedings of the Twelfth Marcel Grossmann Meeting on General Relativity], World Scientific (2010). 